Chen, K., Xue, F., and Lu, W. (2017). "Development of 3D building models using multi-source data: A study of high-density urban area in Hong Kong." In: LC3 2017: Volume I - Proceedings of the Joint Conference on Computing in Construction (JC3), July 4-7, 2017, Heraklion, Greece, pp. 609-616. DOI: https://doi.org/10.24928/JC3-2017/0252.

\title{
DEVELOPMENT OF 3D BUILDING MODELS USING MULTI-SOURCE DATA: A STUDY OF HIGH-DENSITY URBAN AREA IN HONG KONG
}

\author{
Ke Chen ${ }^{1}$, Fan Xue ${ }^{2}$, and Weisheng $\mathrm{Lu}^{3}$
}

\begin{abstract}
There has been a world-wide interest in using 3D building models to support urban planning, design, and management. For high-density urban areas with complex topographic conditions, how to fast develop such models with adequate accuracy remains a challenge, particularly when a single data source is utilized. This study seeks to develop such quality models by integrating multi-source data including the topographic map and LiDAR data. A total of 1,361 high-rise buildings located in Hong Kong are chosen as the subjects. The topographic map is used to develop a level of detail 1 (LoD1) model of each building. The LiDAR data is then used to fine-tune building shapes, in particular roofs, so that more accurate models with LoD2 can be derived. It is found that the integration of topographic map and LiDAR data can improve the completeness and accuracy of building models. The speed and computations to operate the integration are acceptable. Future studies are recommended to achieve LoD3 building models by utilizing other data sources (e.g., semantic information of individual buildings, and points of interest).
\end{abstract}

Keywords: 3D building models, LiDAR data, topographic map, high-density urban area.

\section{INTRODUCTION}

Developing virtual 3D city models is an important part of urban scene reconstruction. A virtual 3D city model represents urban spatial data including sites, infrastructure, buildings and landscape elements. Among all these ingredients, the models of buildings are considered of great importance, which can support various urban planning, design, and management practices including environment management, automatic emergency response, and location-based services (LBS) (Gröger and Plümer 2012; Sun and Salvaggio 2013). Traditionally, buildings in a city model were represented as single polyhedrons (e.g., a cube or cuboid) that have little information of building elements such as roofs and other assemblies. People are increasingly unsatisfactory with the rough representation and seek to capture more detailed building geometry.

There has been a significant progress achieved in this field, though, it remains a challenge to develop 3D models of buildings located in complex topographic conditions such as high-density urban areas. The accuracy of the collected data for developing building models generally would be constrained by the complexity of a dense environment. Additionally, with respect to numerous buildings in a high-density urban area, the manual

PhD Candidate, iLab, Faculty of Architecture, the University of Hong Kong, Hong Kong, leochen@connect.hku.hk

2 Research Assistant Professor, iLab, Faculty of Architecture, the University of Hong Kong, Hong Kong, xuef@hku.hk

3 Associate Professor, iLab, Faculty of Architecture, the University of Hong Kong, Hong Kong, wilsonlu@hku.hk 
editing of models can be extremely time-consuming and error-prone. Hence, it is crucial to introduce automatic approaches for generating such building models amid a 3D virtual city model.

Researchers have proposed various methods to automatically or semi-automatically extract the boundary, roof shape, or even the entire block of a building from a singlesource data (e.g., Sampath and Shan 2010; Sun and Salvaggio 2013; Li et al. 2014). With the advent of data-acquisition technologies such as laser scanning and photogrammetry, it becomes promising to use multi-source data for developing 3D building models in highdensity urban areas, for ultimately overcoming challenges as pinpointed above.

This study aims to develop a strategy of developing 3D models of densely-distributed buildings by integrating topographic map and LiDAR data. A total of 1,819 high-rise buildings located in Hong Kong (HK) Island are chosen as the research subjects. HK Island is one of the most famous high-density urban areas, where the current population density is 7,050 persons per square kilometre (Worldometers 2017).

Following contents start with a review of the state-of-the-art studies on 3D building model generation in Section 2. Section 3 introduces the modelling strategy proposed by this research, followed by the results as described in Section 4. Section 5 concludes this paper and highlights future research directions.

\section{LITERATURE REVIEW}

It has been widely recognized that many shortcomings exist in using the single-source data for 3D building reconstruction. When interpreting the aerial images for developing a $3 \mathrm{D}$ building model, a great amount of manual work is required to ensure the interpretation accuracy (Haala and Kada 2010). Using the airborne LiDAR can enhance the automatic level of 3D reconstruction. However, the quality of the developed models is restricted by the resolution that is often at one-meter level (Cheng et al. 2011).

Drawbacks of using single-source data give rise to the focus on the use of multi-source data. For example, Remondino (2011) used the coloured point cloud generated from the photogrammetry and laser scanning to develop the models of building heritage. Ding et al. (2008) proposed a registration method for applying the texture images to 3D geometry model obtained from LiDAR. Based on the LiDAR data and aerial images, Habib et al. (2010) developed 3D building models. In their models, however, building roofs are bounded by simple straight lines which cannot reflect the actual shapes. Approaches to improve the integration of LiDAR data with aerial images were subsequently proposed by Cheng et al. (2011), Awrangjeb et al. (2013), and several other scholars. For instance, Siddiqui et al. (2016) proposed a gradient-based building extraction (GBE) method to develop building models from LiDAR and photogrammetry. These studies are indeed significant as they contribute to the synergistic use of the multi-source data for building model generation.

Being innovative the existing 3D building reconstruction methods are, it is surprisingly rare for studies on generating quality models of densely-distributed buildings in urban areas. In these areas, not only more complex topographic conditions are in front during data collection, but the reconstruction of numerous buildings cannot be efficiently attained by manual editing. The present research thus is timely as it addresses these specified difficulties. 


\section{Using Multi-SOURCE DATA For 3D BUILDING MODELLING}

\subsection{The Strategy}

To develop 3D models of densely-distributed buildings, this research uses two datasets including the topographic map and LiDAR data. These two datasets cover the same set of 1,361 buildings in the same region in HK Island. The first step of the model development is to use the topographic map to reconstruct level of detail 1 (LoD1) models of the target buildings (See Kolbe et al. [2005] for the definitions of LoD). Next, the LiDAR data that contains more details such as building roofs is used to enrich the LoD1 models into LoD2 models with higher information completeness and accuracy. The integration of the topographic map and LiDAR data is necessary as both datasets have their own merits and defects. For the topographic map, the contained information is only basic to create the 'simple outer shell' of buildings. For the LiDAR data, it may not able to cover lower levels of some buildings since the laser light when scanning a city is often blocked by trees and surrounding buildings in a high-density urban area.

\subsection{Developing LoD1 Building Models}

The digital topographic map - iB1000 - was provided by the Lands Department (LD) of Hong Kong Government. It is in FGDB format with a scale of 1:1000. The coordinates of the map are in Hong Kong 1980 Grid, and heights are in meters above the Hong Kong Principal Datum. Figure 1 shows the data contained in the iB1000.

\begin{tabular}{|c|c|}
\hline $\begin{array}{l}\text { Buildings } \\
\text { Hydrography } \\
\text { LandCover } \\
\text { PlacesOfinterest } \\
\text { Relief } \\
\text { Transportation } \\
\text { Utilities } \\
\text { ADDRESS } \\
\text { ADDRESSRELATEBUILDINGSITE }\end{array}$ & 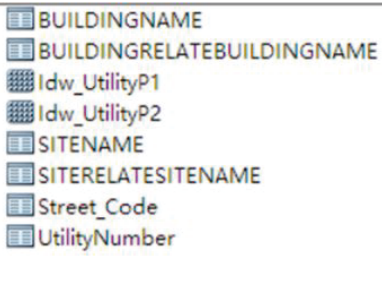 \\
\hline
\end{tabular}

Figure 1: Data contained in the iB1000

As shown in Figure 2, areas coloured in green show the land surfaces covered by buildings, and the blue lines show the boundary of building structures. Besides, iB1000 provides other types of data related to each building, which are the building ID, shape, shape area, type of building block, base level, roof level, data source, and status.

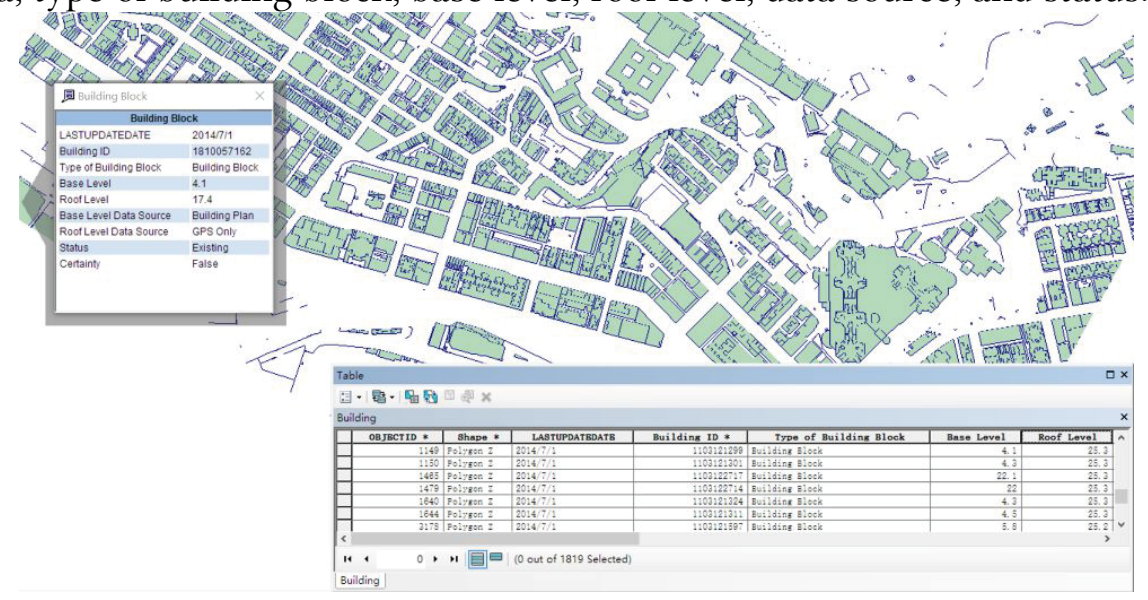

Figure 2: Data of 1,361 buildings in the selected region 
Among all the available data, the 'base level' and 'roof level' of individual buildings are used to developing the 3D building models. These two data strings, as indicated in the iB1000, are derived from the original building plan, photogrammetry, and topographic survey. By defining the base height and using the extrusion function provided by ArcScene ${ }^{\circledR}$ (Esri n.d.), a preliminary 3D reconstruction of 1,361 buildings can be generated as shown in Figure 3.

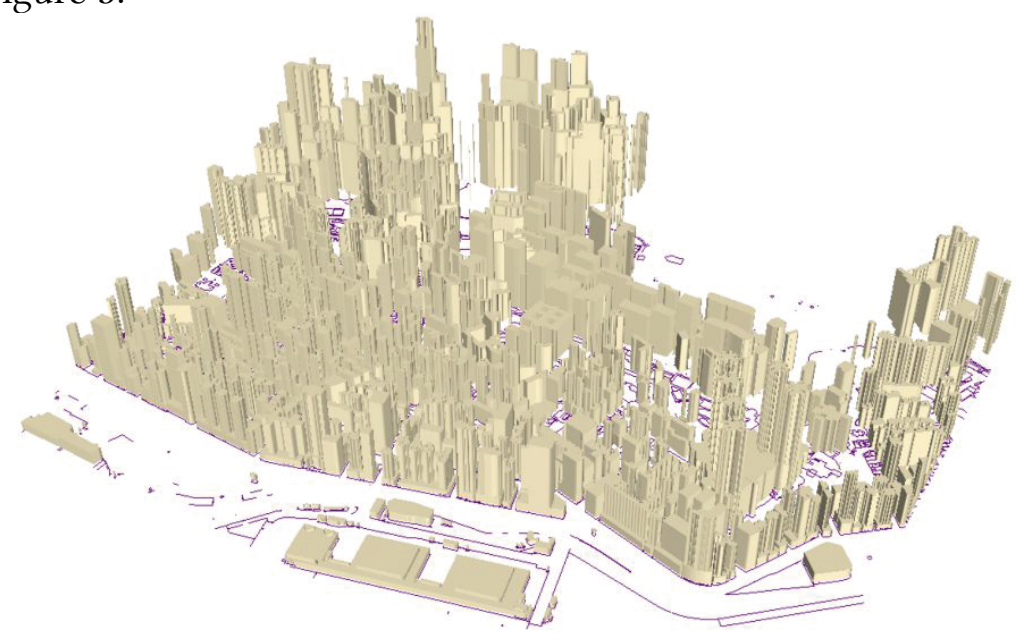

Figure 3: Preliminary 3D reconstruction of 1,361 buildings in the selected region

Based on the data from the topographic map, LoD1 models of the target buildings in the subject area can be developed within half an hour, which is nonetheless not satisfactory in completeness and accuracy. Some building elements, in particular for the roofs, cannot be properly modelled due to a lack of data in the topographic map. The LiDAR data is used in order to improve the model completeness and accuracy by modelling the missing building elements.

\subsection{Integrating Topographic Map with LiDAR Data}

\subsubsection{LiDAR Data}

The LiDAR data, in the form of point clouds, was provided by the Civil Engineering and Development Department (CEDD) of Hong Kong. Figure 4 is an excerpt of the LiDAR data, which contains buildings, trees, roads, and other objects of the subject area. As the data was collected between 1 Dec. 2010 and 8 Jan. 2011, the LiDAR data of new buildings constructed after 2011 was thus unavailable.

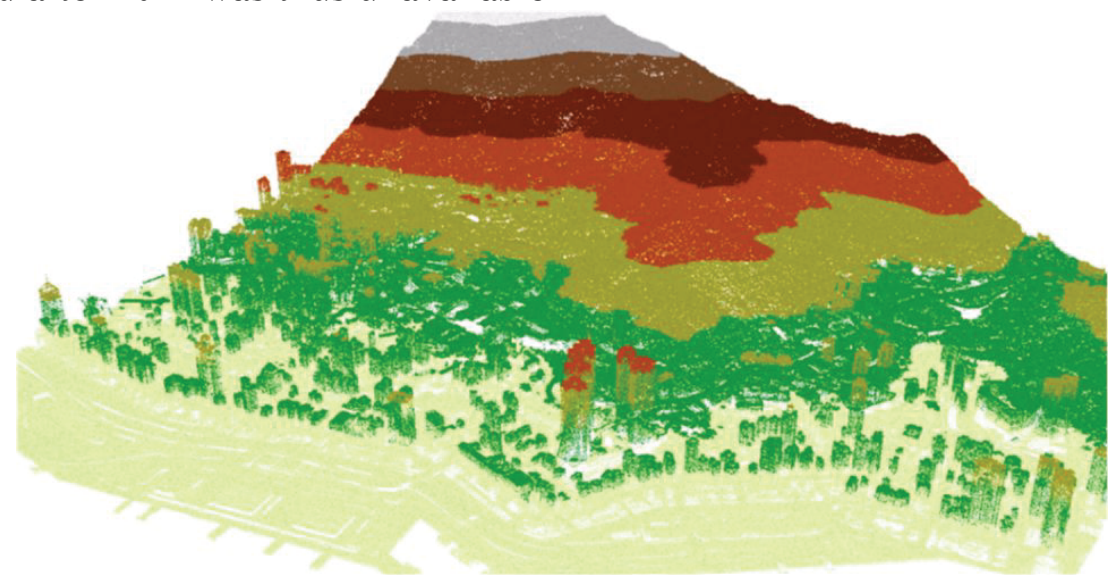

Figure 4: Parts of the LiDAR data of the selected region 
As the LiDAR data contains both buildings and other objects in the subject area, the first step is to separate the point clouds of buildings from those of trees, terrain, and others features. This is done through processing the LiDAR data in 3Dsurvey ${ }^{\circledR}$. After processing, the LiDAR data of target buildings is then adjusted to the same coordination system of the topographic map (see Figure 5).

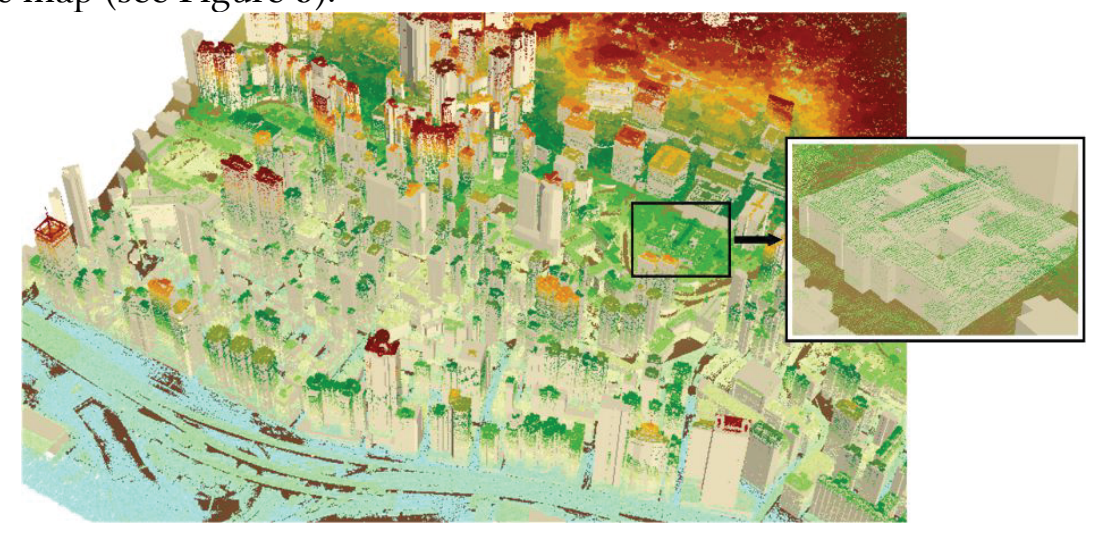

Figure 5: Placing the topographic map and LiDAR data into the same coordination system

\subsubsection{Developing LoD2 Building Models by Footprint Guided Plane Detection}

In this paper, we develop a fully automatic method to generate models of roofs and assemblies, such as foot bridges, squares, and slopes, from LiDAR data. The perpendicular line, or the parallel line, of the longest edge of a building footprint is used as a guide line to enhance plane detection of RANSAC (RAndom SAmple Consensus). RANSAC is well known as an effective heuristic search method in finding geometric primitives such as planes and spheres.

The whole process consists of a few steps. First, we identify a guide line of each building footprint (see Figure 6). Then, the LiDAR data is segmented into subsets of roofs and assemblies through the areas and heights, e.g., Figure 7(a). Schnabel et al.'s (2007) improved RANSAC method is employed to identify planes from the point clouds of roofs and assemblies, as shown in Figure 7(b). The planes are snapped to a set of guided directions following the procedure described in Figure 8. Finally, the planes were projected to the topographic map to generate volumetric roofs and assemblies (see Figure $7[\mathrm{c}]$ ).

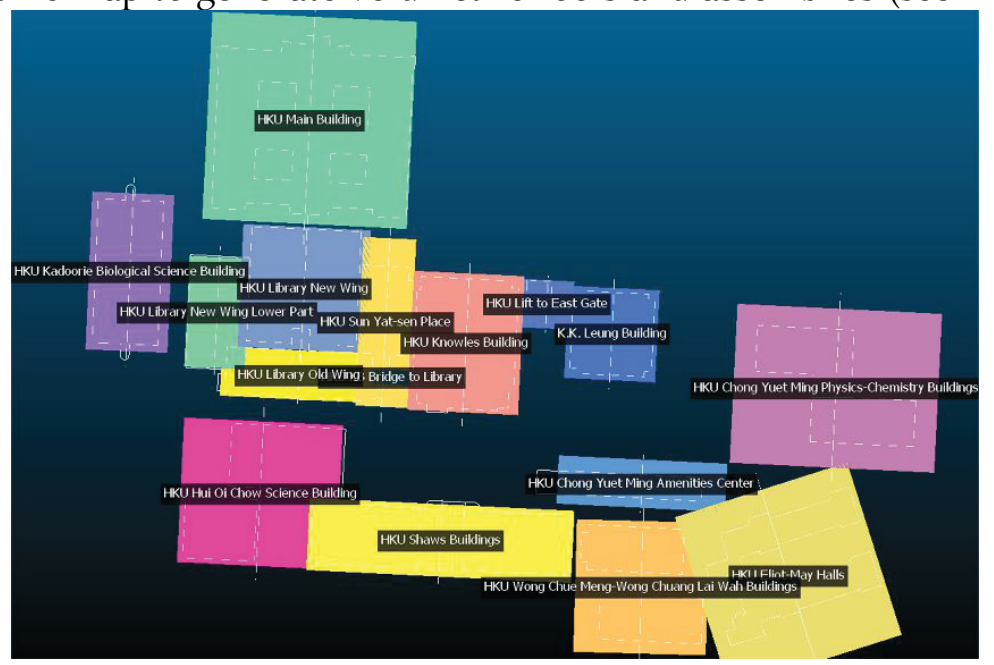

Figure 6: Guide lines identified from building footprints in a sample area of the topographic map 


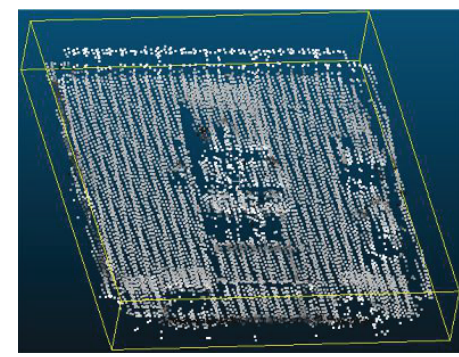

(a) LiDAR data of the roof of Knowles Building at HKU

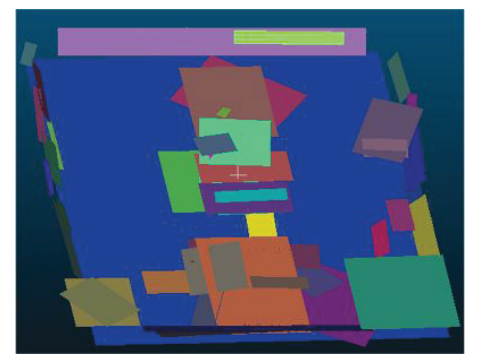

(b) Planes detected by Schnabel et al. (2007)'s RANSAC

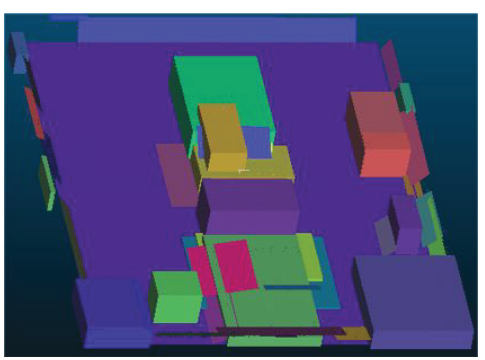

(c) Aligned to the guide line of Knowles Building at HKU

Figure 7: An example of using guide lines of building footprints to rectify geometry detection

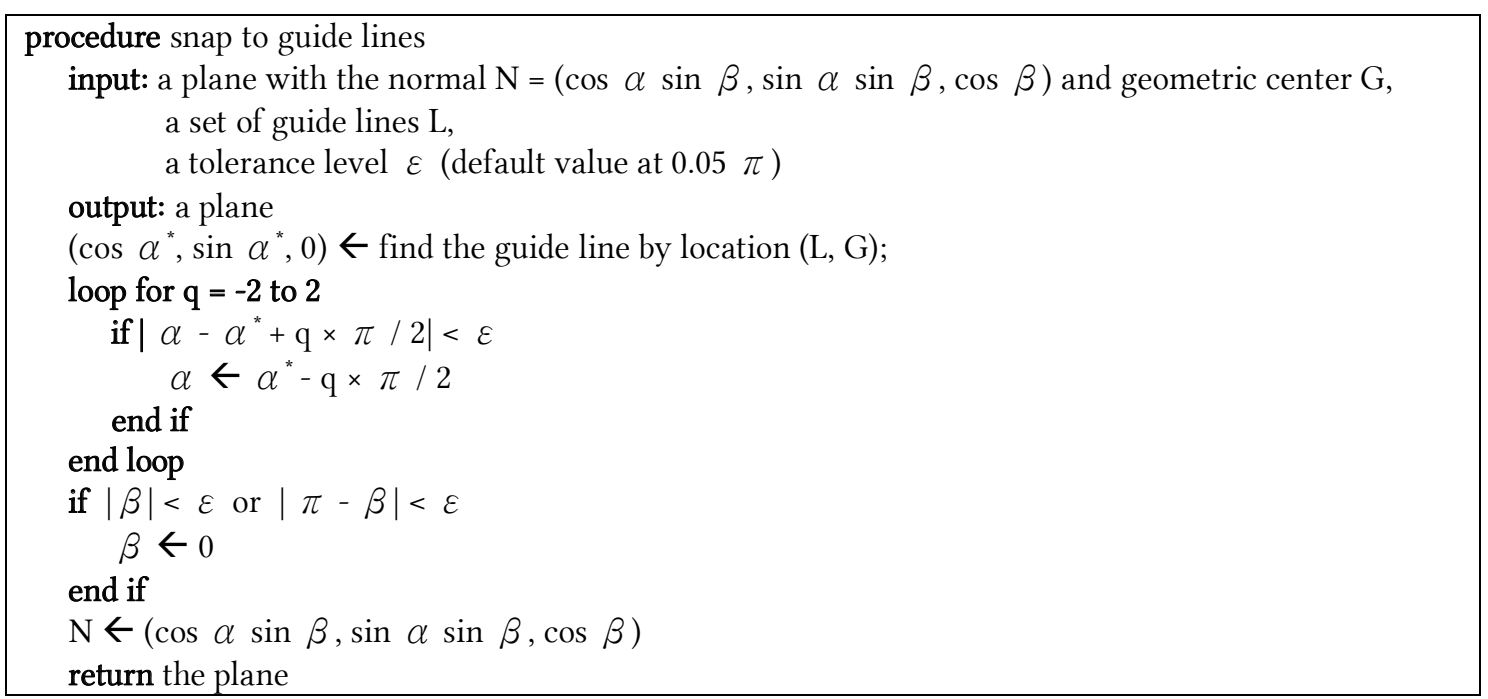

Figure 8: The pseudo code of the line-guided plane correction procedure for building surface models

\section{RESULTS AND DisCUSSIONS}

By following the strategy as introduced in Section 3, LoD2 models of buildings in the selected region can be developed. Examples of the generated models are shown in Figure 9.

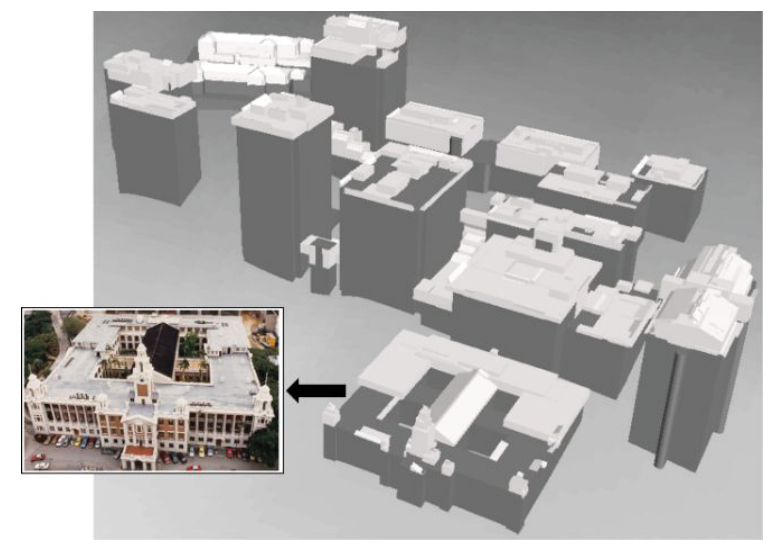

Figure 9: Examples of the generated LoD2 building models (photo retrieved from http://dragageshk.com/) 
A visual inspection identified that the developed models have an acceptable level of completeness and accuracy. However, two problems have also been noted during the modelling process. Firstly, modelling the irregular-shaped roofs (e.g. a dome) and building assemblies requires much more time than modelling those with regular-shaped. This problem can be partially solved if the pre-existing knowledge of building characteristics is available. The second problem involves the time difference between the collection of the two datasets. As mentioned above, most of the data contained in the topographic map was collected in 2014, while the LiDAR data was collected between 2010 and 2011. Consequently, some building elements could be missing in the final 3D building models.

The LoD of the developed models can be further increased to a higher level, i.e., LoD3, by adding more detailed exterior features including openings and wall structures. This requires data from other sources such as the aerial images or the photos from the Internet that can provide the texture of the building surfaces. Besides, the advancement of machine learning (ML) and computer vision (CV) will provide power tools to automatically extract the semantic information of all kind of building surfaces, building elements, and points of interest.

\section{CONCLUSIONS}

Developing 3D building models for reconstructing the city scene has become an important instrument for urban planning activities. Various methods have been proposed to extract or model buildings from aerial images or LiDAR data. When it comes to the high-density urban areas, the situation becomes much more complicated, where the accuracy of models developed from single-source data might become questionable. It is thus important to take advantages of multi-source data to automatically develop 3D building models as accurate as possible.

In this study, a strategy is proposed which integrates the topographic map and LiDAR data for 3D building reconstruction. The topographic map is used to model the 'outer shell' of each building. Besides, an innovative method is developed for modelling the missing building elements from the LiDAR data. The results of this study confirm using multisource data can improve the overall completeness and accuracy of the developed models.

Future research will be conducted in mainly two directions. Firstly, the dimensions of the developed building models will be compared with the ground truth. Secondly, data from more sources will be integrated with the topographic map and LiDAR data to develop LoD3 building models which contains rich semantic information.

\section{REFERENCES}

Awrangjeb, M., Zhang, C., and Fraser, C. S. (2013). Automatic extraction of building roofs using LIDAR data and multispectral imagery. ISPRS J. Photogramm. Remote Sens., 83, pp. 1-18, doi:10.1016/j.isprsjprs.2013.05.006, 1-18.

Cheng, L., Gong, J., Li, M., and Liu, Y. (2011). 3D building model reconstruction from multi-view aerial imagery and lidar data. Photogramm. Eng. Remote Sens., 77(2), pp. 125-139, doi:10.14358/PERS.77.2.125, 125-139.

Ding, M., Lyngbaek, K., and Zakhor, A. (2013). Automatic registration of aerial imagery with untextured 3d lidar models. In Computer Vision and Pattern Recognition 2008, IEEE, pp. 1-18, doi:10.1109/CVPR.2008.4587661, 1-18. 
Esri (n.d.). Extrude features to 3D symbology. Available at:http://pro.arcgis.com/en/proapp/help/mapping/layer-properties/extrude-features-to-3d-symbology.htm [Accessed 6 Jan. 2017].

Gröger, G., and Plümer, L. (2012). Citygml - interoperable semantic 3d city models. ISPRS J. Photogramm. Remote Sens., 71(7), pp. 12-33, doi:10.1016/j.isprsjprs.2012.04.004, 1233.

Haala, N., and Kada, M. (2010). An update on automatic 3D building reconstruction. ISPRS J. Photogramm. Remote Sens., 65(6), pp. 570-580, doi:10.1016/j.isprsjprs.2010.09.006, 570-580.

Habib, A. F., Zhai, R., and Kim, C. (2010). Generation of complex polyhedral building models by integrating stereo-aerial imagery and lidar data. Photogramm. Eng. Remote Sens., 76(5), pp. 609-623, doi:10.14358/PERS.76.5.609, 609-623.

Kolbe, T. H., Gröger, G., and Plümer, L. (2005). CityGML: Interoperable access to 3D city models. In: P. van Oosterom, S. Zlatanova, E. M. Fendel, ed., Geo-information for Disaster Management. Springer Berlin Heidelberg, pp. 883-899, doi: 10.1007/3-54027468-5_63.

Li, J., Huang, W., Shao, L., and Allinson, N. (2014). Building recognition in urban environments: A survey of state-of-the-art and future challenges. Inform. Sciences, 277, pp. 406-420, doi:10.1016/j.ins.2014.02.112, 406-420.

Remondino, F. (2011). Heritage recording and 3D modeling with photogrammetry and 3D scanning. Remote Sens., 3(6), pp. 1104-1138, doi: 10.3390/rs3061104, 1104-1138.

Sampath, A., and Shan, J. (2010). Segmentation and reconstruction of polyhedral building roofs from aerial lidar point clouds. IEEE Trans. Geosci. Remote Sens., 48(3), pp. 15541567, doi:10.1109/TGRS.2009.2030180, 1554-1567.

Schnabel, R., Wahl, R., and Klein, R. (2007). Efficient RANSAC for point-cloud shape detection. Comput. Graph. Forum, 26(2), pp. 214-226, doi: 10.1111/j.14678659.2007.01016.x, 214-226.

Siddiqui, F. U., Teng, S. W., Awrangjeb, M., and Lu, G. (2016). A Robust Gradient Based Method for Building Extraction from LiDAR and Photogrammetric Imagery. Sensors, 16(7), pp. 1110, doi:10.3390/s16071110, 1110.

Sun, S., and Salvaggio, C. (2013). Aerial 3D building detection and modeling from airborne LiDAR point clouds. IEEE J. Sel. Topics Appl. Earth Observ. in Remote Sens., 6(3), pp. 1440-1449, doi:10.1109/JSTARS.2013.2251457, 1440-1449.

Worldometers (2017). Hong Kong Population, Available at: http://www.worldometers.info/world-population/china-hong-kong-sar-population/ [Accessed on 15 Jan. 2017] 